\title{
Magnetic resonance imaging in mood disorders: a bibliometric analysis from 1999 to 2020
}

\author{
Mingzhou Gao ${ }^{1,3} \cdot$ Hui Sun ${ }^{2} \cdot$ Xunshu Cheng ${ }^{1} \cdot$ Dongmei Gao ${ }^{1,3} \cdot$ Mingqi Qiao $^{1,3}$
}

Received: 24 January 2021 / Accepted: 7 April 2021 / Published online: 6 May 2021

(c) The Author(s) 2021

\begin{abstract}
Objective Globally, mood disorders are highly prevalent, and are associated with increased morbidity and mortalities. Magnetic resonance imaging is widely used in the study of mood disorders. However, bibliometric analyses of the state of this field are lacking.

Methods A literature search in the web of science core collection (WoSCC) for the period between 1945 and 2020 returned 3073 results. Data extracted from these publications include, publication year, journal names, countries of origin, institutions, author names and research areas. The bibliometric method, CiteSpace V and key words analysis were used to visualize the collaboration network and identify research trends, respectively.

Results Since it was first reported in 1999, the use of magnetic resonance imaging in studies on mood disorders has been increasing. Biological psychiatry is the core journal that has extensively published on this topic, while the UNIV PITTSBURGH, USA, has the highest published papers on this topic. Keyword analysis indicated that studies on depression, bipolar disorders, and schizophrenia, with a focus on specific brain regions, including amygdala, prefrontal cortex and anterior cingulate cortex are key research topics.

Conclusion Brain structure and network, sex differences, and treatment-associated brain changes are key topics of future research.
\end{abstract}

Keywords Mood disorders $\cdot$ Depression $\cdot$ Anxiety $\cdot$ Citespace $\cdot$ f-MRI

\section{Introduction}

Mood disorders are a class of psychiatric conditions that alter moods, energy and motivation. This group of disorders include depression, bipolar disorder, premenstrual

Mingzhou Gao and Hui Sun have contributed equally to this work and should be considered co-first authors.

Dongmei Gao

gcy_112@163.com

$\triangle$ Mingqi Qiao qmingqi@163.com

1 College of Traditional Chinese Medicine, Shandong University of Traditional Chinese Medicine, Jinan, Shandong Province, China

2 School of Pharmaceutical Sciences, South-Central University for Nationalities, Wuhan, Hubei Province, China

3 Research and Innovation Team of Emotional Diseases and Syndromes in Shandong University of Traditional Chinese Medicine, Jinan, Shandong Province, China syndrome, premenstrual dysphoric disorder, and the autism spectrum of disorders among others. Globally, mood disorders affect $>300$ million people with devastating consequences $[1,2]$. Previous studies have reported the clinical prevalence of mood disorders [3, 4], with children, adolescents, elderly people and patients with neurological conditions being prone to depressive disorders $[5,6]$. The pathophysiology of mood disorders is complex and not properly elucidated $[7,8]$. Moreover, it is influenced by genetic and environmental factors that may disrupt neurotransmitter homeostasis $[7,8]$. Therefore, mood disorders may result from negative feelings that leave a lasting influence on mental processes associated with perception, cognition and motor systems $[9,10]$. In affective disorders, brain morphological alterations largely occur on the fronto-limbic cortex, hippocampus and amygdala. These structures modulate an individual's emotional and cognitive function, and mood disorder manifestation [11]. Neuroimaging has revealed that brain networks that control emotional behaviors can also influence mood disorder pathophysiology [12]. Advances 
in in vivo neuroimaging techniques have, therefore, greatly improved our understanding of mood disorder pathophysiology [13].

There are various clinical imaging techniques, such as PET, DTI [14], ASL [15], and 1H-MRS [16]. However, magnetic resonance imaging (MRI), one of the neuroimaging techniques that are widely used in neuroscience to visualize neural activities, and to identify mental disease biomarkers [17] has unique advantages. In particular, MRI is now widely used to evaluate mood disorders $[18,19]$, such as depression [20] and premenstrual dysphoric disorder [21]. Advances in this technology will enable quantification of biochemical components of a functioning brain that are not detectable [22]. However, despite progress in the use of MRI to study mood disorders, accompanying bibliometric analysis is lacking.

Bibliometrics is a statistical analysis and quantitative tool for identifying research hotspots and frontiers in a particular field within a short time by extracting quantitative information on study distributions by country/region, institution, author, and journal [23]. Now, bibliometric methods in Citespace have been widely used in medical fields, such as acupuncture [24] and regenerative medicine [25]. The CiteSpace software was invented by Professor Chaomei Chen in early 2004. It is based on scientometrics and knowledge visualization, and is effective in mining scientific literature for critical information. It is used to establish co-occurrence network maps of authors, keywords, institutions, countries, and subject categories as well as co-citation networks of cited authors, cited references, and cited journals. For any discipline of interest, CiteSpace can greatly inform on basic knowledge, identify seminal studies in the area, uncover current research themes, and contextualize research advances [26].

Therefore, this study aimed at comprehensively analyzing the current status and developing trends of related references derived from Web of Science database from 1999 to 2020 in global research on mood disorders by magnetic resonance imaging through a bibliometric and visual analysis.

\section{Materials and methods}

\section{Data collection and screening}

Online literature search was performed in web of science core collection (WoSCC). To minimize bias from daily database updates, the search was done on the same day (April 29th, 2020) using the following search terms: $(\mathrm{TS}=($ Disorder, Mood $)$ OR TS $=($ Disorders, Mood $)$ OR $\mathrm{TS}=($ Mood Disorder $)$ OR TS $=($ Affective Disorders $)$ OR TS $=($ Affective Disorder $)$ OR TS $=($ Disorder, Affective) OR TS $=($ Disorders, Affective $)$ ) AND ((Functional
MRI) OR TS: (Functional MRIs) OR TS: (MRIs, Functional) OR TS: (Functional Magnetic Resonance Imaging) OR TS: (Magnetic Resonance Imaging, Functional) OR TS: (Spin Echo Imaging) OR TS: (Echo Imaging, Spin) OR TS: (Echo Imagings, Spin) OR TS: (Imaging, Spin Echo) OR TS: (Imagings, Spin Echo) OR TS: (Spin Echo Imagings) OR TS: (Imaging, Magnetic Resonance) OR TS: (NMR Imaging) OR TS: (Imaging, NMR) OR TS: (Tomography, NMR) OR TS: (Tomography, MR) OR TS: (MR Tomography) OR TS: (NMR Tomography) OR TS: (Steady-State Free Precession MRI) OR TS: (Steady-State Free Precession MRI) OR TS: (Zeugmatography) OR TS: (Imaging, Chemical Shift) OR TS: (Chemical Shift Imagings) OR TS: (Imagings, Chemical Shift) OR TS: (Shift Imaging, Chemical) OR TS: (Shift Imagings, Chemical) OR TS: (Chemical Shift Imaging) OR TS: (Tomography, Proton Spin) OR TS: (Proton Spin Tomography) OR TS: (Magnetization Transfer Contrast Imaging) OR TS: (MRI Scans) OR TS: (MRI Scan) OR TS: (Scan, MRI) OR TS: (Scans, MRI) OR TS: (fMRI) OR TS: (MRI, Functional)) AND Literature type: (Article), Indices $=$ SCIEXPANDED, SSCI, CCR-EXPANDED, IC time span = all years. Only published articles were selected, and publication language was unrestricted (Fig. 1).

Through descriptive analysis, we characterized the selected studies by publication year, country, journal, and authors and then constructed knowledge maps using the CiteSpace software (version 5.3.R4). Finally, citation growth rate or keywords were evaluated using burst in CiteSpace V.

\section{Results and discussion}

\section{General information of publications}

Due to its quality curation of research studies, WoS is a reliable source of studies for bibliometric analysis of scientific literature [27, 28]. Our first round of literature search found 54,346 and 331,162 reports on mood disorders and MRI, respectively. Among them, a total of 3073 original publications involving MRI in mood disorder studies were selected for further analysis. There was an increase in the number of publications on this topic between 1999 (28) and 2019 (236). Notably, in the last 4 months of 2020, 66 original studies were published (Fig. 2a). Citation scores of these studies for the $1999(16)$ and $2019(14,915)$ years exhibited a significant increase. An increase in citation scores by 4039 resulted in a total score of 142,188, with an h-index of 166 and on average, 46.27 references per item (Fig. 2b). These results indicate that MRI is increasingly becoming popular as a mood disorder research tool. 


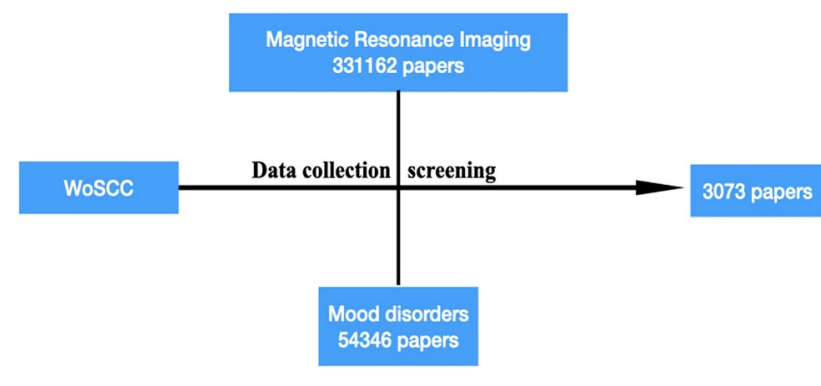

Fig. 1 Schematic diagram of data collection and screening methods

\section{Funding analysis}

Adequate research funding is crucial for scientific advancement and development of future researchers [29]. Research grant applications is critical in advancing one's academic career [30]. The 3073 published studies included in this study were supported by a total of 2976 grants. The top 10 funders were United States department of health human services (1000), NIH (999), NIH national institute of mental health (NIMH) (651), national natural science foundation of China (193), Narsad (151), German research foundation (DFG) (148), medical research council (MRC), UK (138), NIH national center for research resources NCRR (114), NIH national institute on drug abuse (NIDA) (76) and Wellcome trust (75) (Table 1). Among the top 10 funders, six are American. Prior to world war II, public research funding was scarce, and was mainly directed at aeronautical and agricultural research. Recently, improved government funding has accelerated basic research, including in China.

\section{Journal analysis}

Journal analysis simplifies identification, tracking, and focus on relevant research in topics of interest. The 3073 studies included in this study are published in 467 different journals. Among them, 185 studies are in biological psychiatry, 175 studies are in the journal of affective disorders, 169 studies are in psychiatry research, 169 studies are in neuroimaging, 103 studies are in neuroimage, 102 studies are in bipolar disorders, 76 studies are in psychological medicine, 72 studies are in human brain mapping, 72 studies are in PLos one, and 69 studies are in the American journal of psychiatry.

CiteSpace journal co-citation analysis identified 1029 highly cited journals and 10,270 network lines. Biological psychiatry was the most cited journal (Fig. 3). Moreover, Biological psychiatry, Neuroimage, American journal of psychiatry, Archives of General Psychiatry, and Journal of neuroscience published many groundbreaking studies that influenced research direction (Tables 2and3). Biological psychiatry is society of biological psychiatry's official journal that is geared towards enhancing research excellence in areas studying the nature, causes, mechanisms, and management of thought, emotion, or behavioral disorders. Biological psychiatry is highly regarded in psychiatric neuroscience and was ranked 2 nd among psychiatric journals in 2019 by google scholar, with an impact factor of 7.27 .

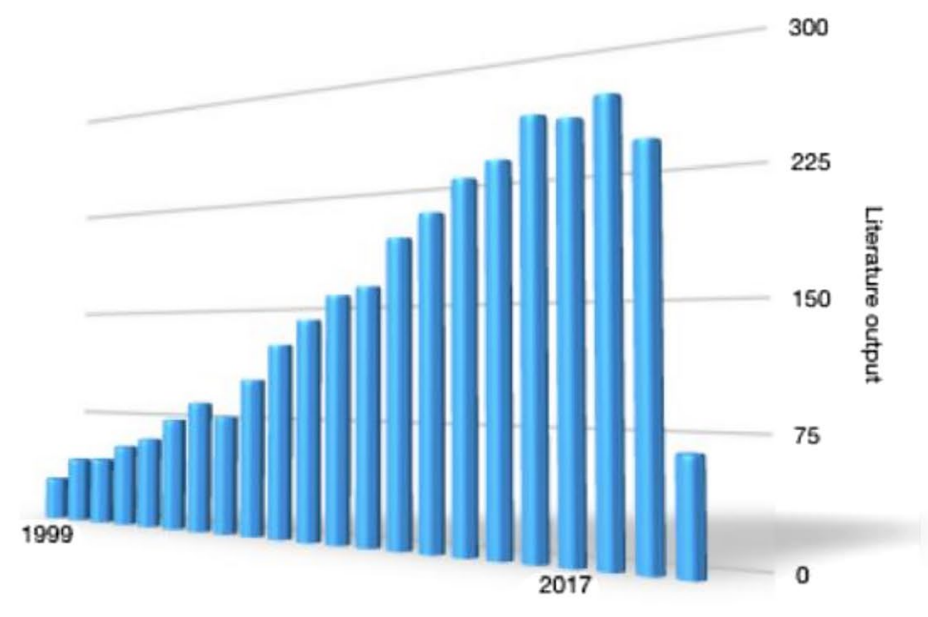

Publishing year

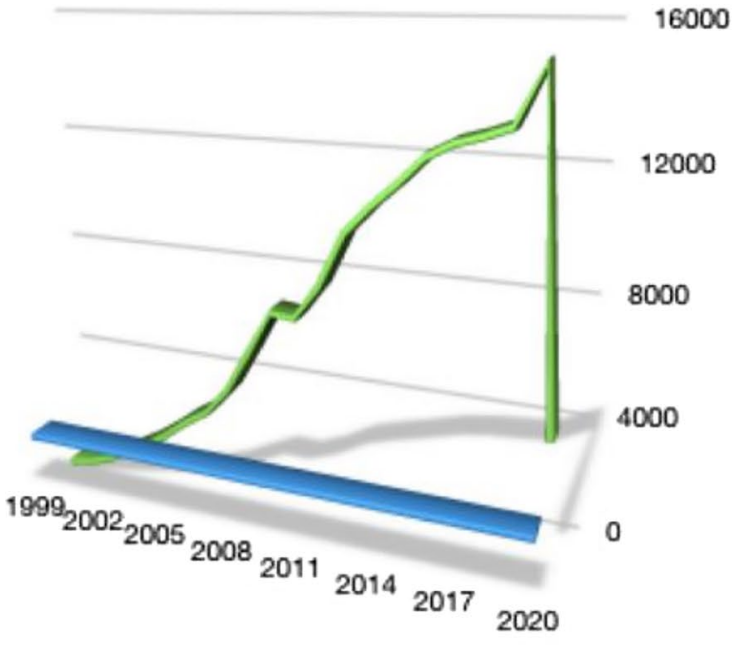

- Publishing year

- Cited frequency

Fig. 2 Literature outputs involving MRI in mood disorder studies and their citation frequency between 1999 and 2020 
Table 1 Funding agencies of the 3073 publications reporting MRI use in mood disorder studies

Fig. 3 Journal co-citation network visualization

\begin{tabular}{lllrc}
\hline Rank & Funding institutions & Country & Records & \% of 3073 \\
\hline 1 & United States Department of Health Human Services & USA & 1000 & 32.541 \\
2 & National Institutes of Health NIH USA & USA & 999 & 32.509 \\
3 & NIH National Institute of Mental Health NIMH & USA & 651 & 21.185 \\
4 & National Natural Science Foundation of China & China & 193 & 6.281 \\
5 & NARSAD & USA & 151 & 4.914 \\
6 & German Research Foundation DFG & German & 148 & 4.816 \\
7 & Medical Research Council UK MRC & UK & 138 & 4.491 \\
8 & NIH National Center for Research Resources NCRR & USA & 114 & 3.71 \\
9 & NIH National Institute on Drug Abuse NIDA & USA & 76 & 2.473 \\
10 & Wellcome Trust & UK & 75 & 2.441 \\
\hline
\end{tabular}

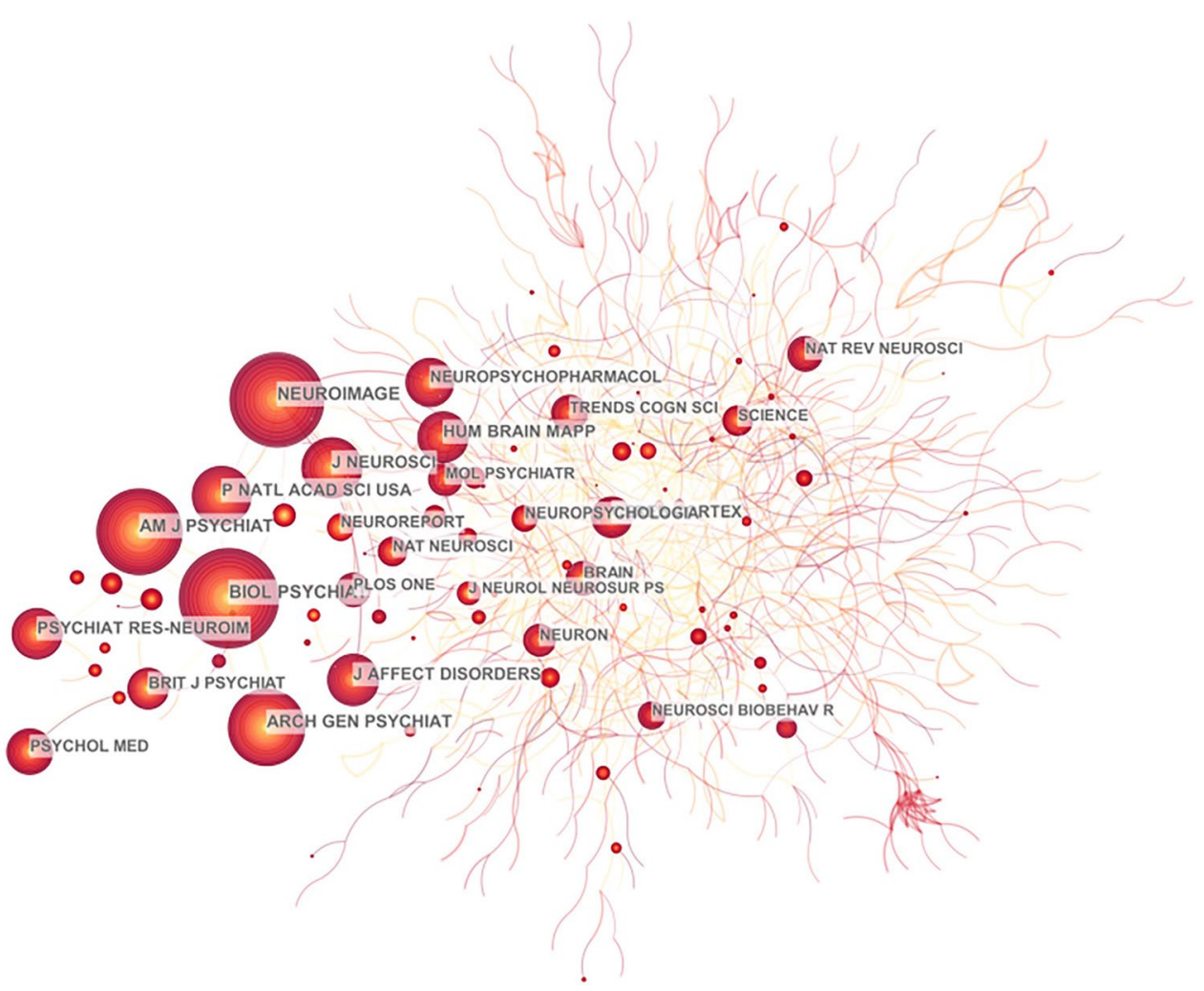

\section{Scientific Research Collaboration Analysis}

Research collaboration is crucial in interdisciplinary research and helps overcome scientific challenges, leading to major research breakthroughs. Research collaboration network refers to the appearance of authors from different research institutions, or countries (regions) in the same paper.

\section{Author analysis}

CiteSpace analysis uncovered 919 nodes (corresponding to 919 prolific authors), and 1642 cooperative lines, indicating intense collaboration between authors (Fig. 4). Node size is directly proportional to the number of publications, while connecting line thickness is directly proportional to the level of collaboration. Phillips ML was the most productive author, with 59 publications, followed by Soares JC (54), Pine DS (48), Brambilla P (44), Drevets WC (42), Leibenluft E (40), Heinz A (33), Keshavan MS (33), Strakowski SM(32) and Birmaher B (31). 
Table 2 Top 10 journals involving MRI use in mood disorder studies

\begin{tabular}{lllll}
\hline Rank & Journal & 记录 & \% of 3073 & CiteScore \\
\hline 1 & Biological Psychiatry & 185 & 6.024 & 7.27 \\
2 & Journal of Affective Disorders & 175 & 5.698 & 4.20 \\
3 & Psychiatry Research Neuro- & 169 & 5.503 & 2.70 \\
$\quad$ imaging & & & \\
4 & Neuroimage & 103 & 3.354 & 6.13 \\
5 & Bipolar Disorders & 102 & 3.321 & 4.27 \\
6 & Psychological Medicine & 76 & 2.475 & 5.77 \\
7 & Human Brain Mapping & 72 & 2.345 & 5.11 \\
8 & Plos One & 72 & 2.345 & 3.02 \\
9 & American Journal of Psychia- & 69 & 2.247 & 6.03 \\
& try & & & \\
10 & Neuropsychopharmacology & 67 & 2.182 & 6.22 \\
\hline
\end{tabular}

Table 3 Top 10 Cited journals related to MRI use in mood disorder studies

\begin{tabular}{lllll}
\hline Rank & Journal & Freq & CiteScore & $\begin{array}{l}\text { Country or } \\
\text { region of publi- } \\
\text { cation }\end{array}$ \\
\hline 1 & Biol Psychiat & 2573 & 7.27 & United States \\
2 & Neuroimage & 2417 & 6.13 & United States \\
3 & Am J Psychiat & 2243 & 6.03 & United States \\
4 & Arch Gen Psychiat & 2057 & 0.00 & United States \\
5 & J Neurosci & 1694 & 5.83 & United States \\
6 & P Natl Acad Sci USA & 1606 & 8.58 & United States \\
7 & Psychiat Res-Neuroim & 1468 & 2.70 & Netherlands \\
8 & Hum Brain Mapping & 1458 & 5.11 & United States \\
9 & J Affect Disorders & 1441 & 4.2 & Netherlands \\
10 & Neuropsychopharmacol & 1353 & 6.22 & England \\
\hline
\end{tabular}

Co-author networks are used to analyze joint research in any field of interest. We found a total of 1029 highly cited authors and 1468 network lines, with Drevets WC being top, with 761 co-authorships (Fig. 5, Table 4).

\section{Country analysis}

CiteSpace analysis identified 57 countries and regions, with 70 collaboration links (Fig. 6), indicating high crosscountry (region) research links. Top countries by publication numbers were USA, Germany, England, China, Canada, Italy, Netherlands, Australia, Brazil and Switzerland. Interestingly, while Germany is the 2nd (481 publications), no German institutions are in the top 10 list of institutions (Fig. 6, Table 5).

\section{Institutional analysis}

CiteSpace analysis identified 40 research institutions and nine collaborations in the co-institute network. Based on the number of publications, the top 10 institutions were University of Pittsburgh, Harvard University, NIMH, King's College London, University of California, Los Angeles, Stanford University, Yale University, University of California San Diego, University of Sao Paulo and McLean Hospital (Fig. 7, Table 5).

\section{Research area analysis}

The studies included covered a total of 50 research fields, including immunology (171), followed by cell biology (65), and biochemistry-molecular biology (60). The top 20 research fields involving MRI use in mood disorders are
Fig. 4 Author journal co-citation network visualization

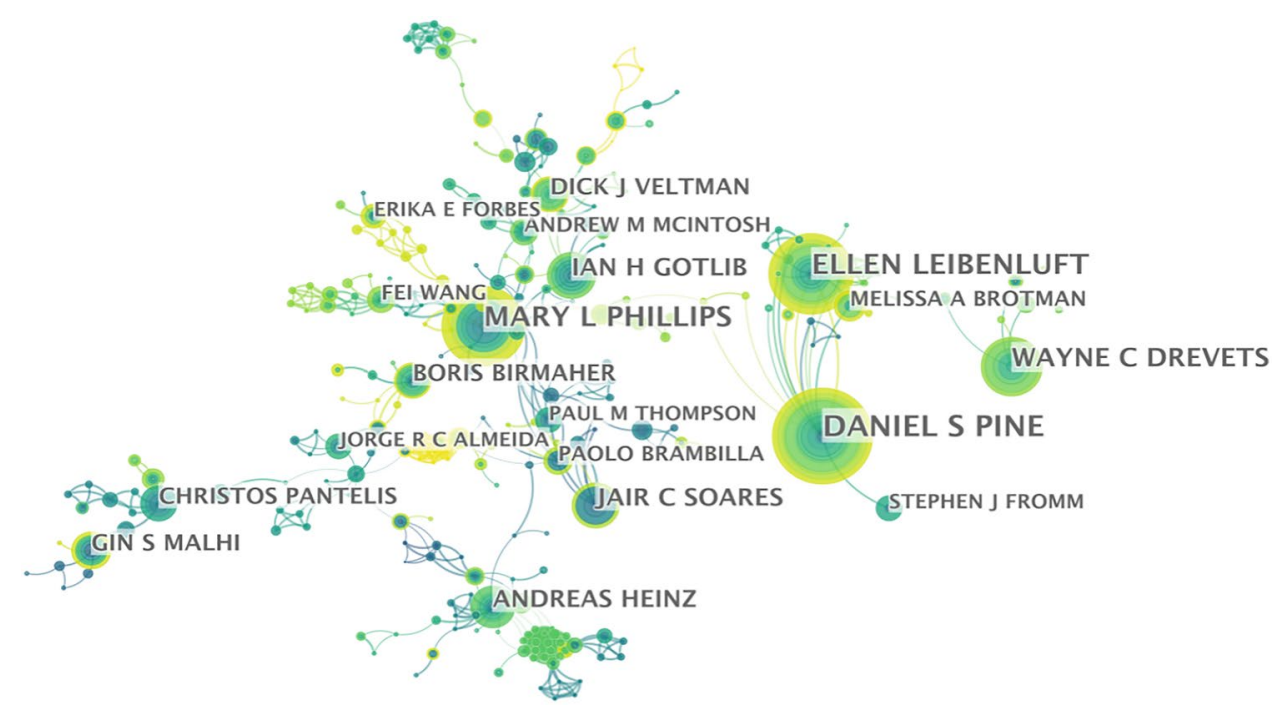




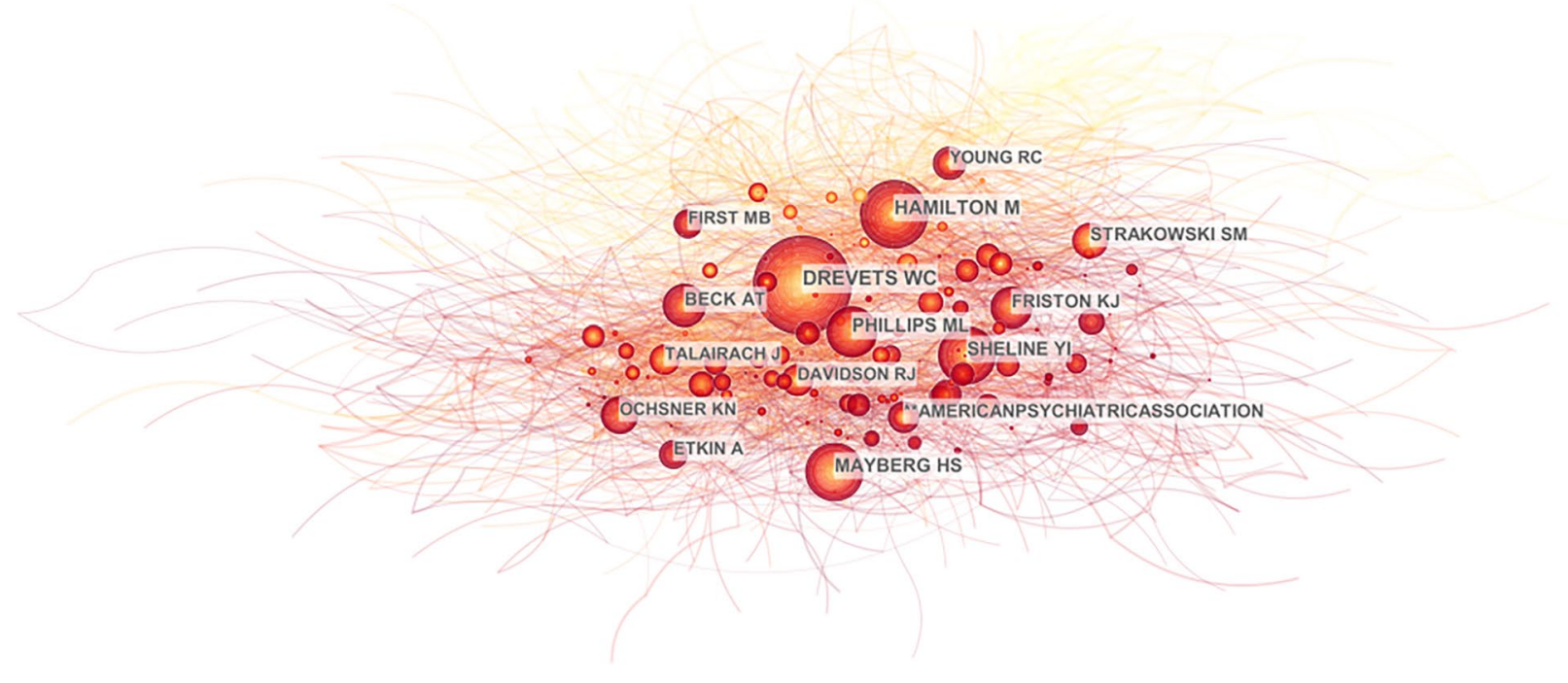

Fig. 5 Author co-citation network visualization

Table 4 Top 10 authors reporting MRI us in mood disorder studies

\begin{tabular}{llllllll}
\hline Rank & Author & Records & $\%$ & Rank & Cited-Author & Freq & Year \\
\hline 1 & Phillips ML & 59 & 1.92 & 1 & Drevets WC & 761 & 1999 \\
2 & Soares JC & 54 & 1.757 & 2 & Hamilton M & 556 & 1999 \\
3 & Pine DS & 48 & 1.562 & 3 & Mayberg HS & 507 & 1999 \\
4 & Brambilla P & 44 & 1.432 & 4 & Sheline YI & 498 & 2000 \\
5 & Drevets WC & 42 & 1.367 & 5 & Phillips ML & 454 & 2003 \\
6 & Leibenluft E & 40 & 1.302 & 6 & Beck AT & 387 & 1999 \\
7 & Heinz A & 33 & 1.074 & 7 & Friston KJ & 386 & 1999 \\
8 & Keshavan MS & 33 & 1.074 & 8 & Strakowski SM & 359 & 1999 \\
9 & Strakowski SM & 32 & 1.041 & 9 & American Psychiat- & 349 & 1999 \\
& & & 1.009 & 10 & Young RC & 311 & 2003 \\
\hline
\end{tabular}

Fig. 6 Country network visualization

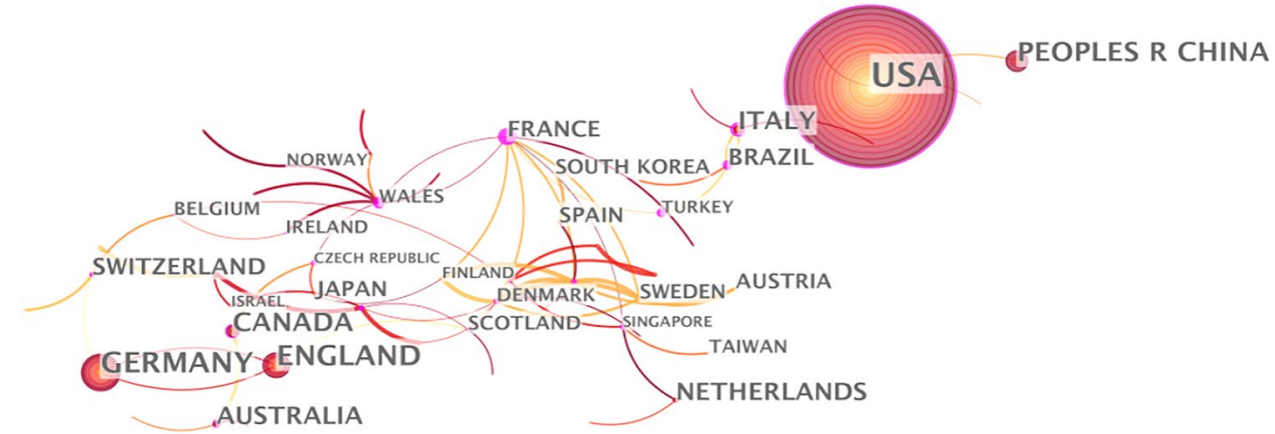

shown in Table 6. Neurosciences, neurology and psychiatry were the main research areas (Table 6).

\section{Cited reference analysis}

Analysis of cited reference co-citation identified 324 nodes and 555 links (Fig. 8). The top 10 cited publications on 
Table 5 Top 10 countries and institutions using MRI in mood disorder research

\begin{tabular}{llcclllll}
\hline Rank & Country/Region & Records & \% of 3073 & Rank & Institution & Country & Records & $\%$ of 3073 \\
\hline 1 & USA & 1538 & 50.049 & 1 & Univ Pittsburgh & USA & 171 & 5.565 \\
2 & Germany & 481 & 15.652 & 2 & Harvard Univ & USA & 139 & 4.523 \\
3 & England & 372 & 12.105 & 3 & NIMH & USA & 129 & 4.198 \\
4 & Peoples R China & 287 & 9.339 & 4 & Kings Coll London & England & 116 & 3.775 \\
5 & Canada & 225 & 7.322 & 5 & Univ Calif Los Angeles & USA & 99 & 3.222 \\
6 & Italy & 194 & 6.313 & 6 & Stanford Univ & USA & 98 & 3.189 \\
7 & Netherlands & 170 & 5.532 & 7 & Yale Univ & USA & 98 & 3.189 \\
8 & Australia & 162 & 5.272 & 8 & Univ Calif San Diego & USA & 74 & 2.408 \\
9 & Brazil & 117 & 3.807 & 9 & Univ Sao Paulo & Brazil & 74 & 2.408 \\
10 & Switzerland & 109 & 3.547 & 10 & Mclean Hosp & USA & 68 & 2.213 \\
\hline
\end{tabular}

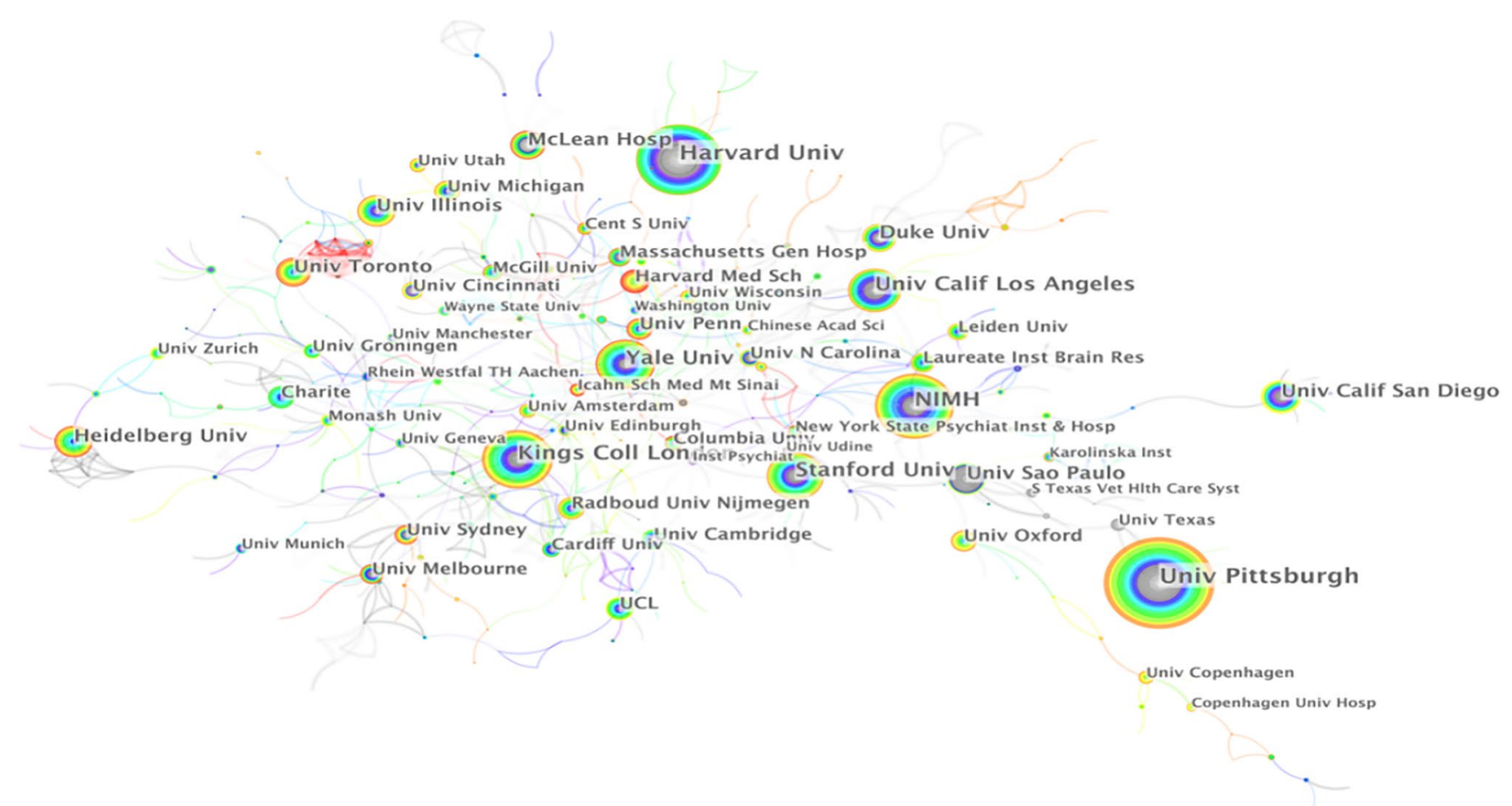

Fig. 7 Institution network visualization

MRI use in mood disorders focused on depression, schizophrenia and post traumatic stress disorder. With a citation frequency of 123, "Brain Structural and Functional Abnormalities in Mood Disorders: Implications for Neurocircuitry Models of Depression", ranked first (Table 7).

\section{Research hotspots and frontiers}

Within any given time period, knowledge maps of keywords co-occurrence reflect hot topics, while trending keywords indicate frontier topics. We used CiteSpace $\mathrm{V}$ to evaluate keywords in titles, abstracts, as well as article keyword sections, and to build keyword co-occurrence networks.

\section{Research hotspots}

Keyword visual knowledge map analysis revealed 130 nodes and 431 links (Fig. 9). With the strongest citation burst keywords being: f-MRI, depression, bipolar disorder, amygdala, mood disorder, prefrontal cortex, brain, major depression, schizophrenia, MRI, emotion, disorder, functional connectivity, meta-analysis, activation, abnormality, major depressive disorder, mood, anterior cingulate cortex, and magnetic resonance imaging (Table 8). 
Table 6 Research areas for the 3073 publications on MRI use in mood disorder studies

\begin{tabular}{llcc}
\hline Rank & Research direction & Records & \% of 3073 \\
\hline 1 & Neurosciences neurology & 1925 & 62.642 \\
2 & Psychiatry & 1741 & 56.655 \\
3 & Psychology & 471 & 15.327 \\
4 & Radiology nuclear medicine medi- & 204 & 6.638 \\
& $\quad$ cal imaging & & \\
5 & Pharmacology pharmacy & 194 & 6.313 \\
6 & Behavioral sciences & 135 & 4.393 \\
7 & Science technology other topics & 130 & 4.23 \\
8 & Pediatrics & 65 & 2.115 \\
9 & Biochemistry molecular biology & 58 & 1.887 \\
10 & Geriatrics gerontology & 42 & 1.367 \\
11 & General internal medicine & 39 & 1.269 \\
12 & Endocrinology metabolism & 37 & 1.204 \\
13 & Research experimental medicine & 35 & 1.139 \\
14 & Substance abuse & 27 & 0.879 \\
15 & Surgery & 20 & 0.651 \\
16 & Rheumatology & 15 & 0.488 \\
17 & Anatomy morphology & 14 & 0.456 \\
18 & Genetics heredity & 13 & 0.423 \\
19 & Physiology & 13 & 0.423 \\
20 & Gastroenterology hepatology & 12 & 0.39 \\
\hline & & &
\end{tabular}

\section{Key diseases of mood disorders}

Individuals experience various emotions, such as sadness, irritation, happiness, and elation. However, people with mood disorders often experience strong emotions, for unusually long periods of time to the extent that emotions negatively impact their daily routines. Depression and bipolar disorders are the main mood disorders. Our analysis revealed 658 and 570 studies on depression and bipolar disorder, respectively. Depression is a significant clinical and public health concern [31] and its pathophysiology has not been clearly elucidated. Environmental as well as cellular and molecular factors trigger functional, and/or structural brain alterations [32] that may contribute to these disorders. Cognitive behavioral therapy is the best-studied psychotherapeutic approach, although its mechanisms of action are unknown [33]. Bipolar disorder is characterized by recurrent episodes of elevated moods and depression, along with changes in activity levels. Bipolar (affective) disorder, (previously manic depressive illness) is extremely difficult to manage [34]. Additionally, major depression and schizophrenia are major research topics. Schizophrenia is a heterogeneous psychiatric disorder with wide-ranging clinical and biological manifestations [35].

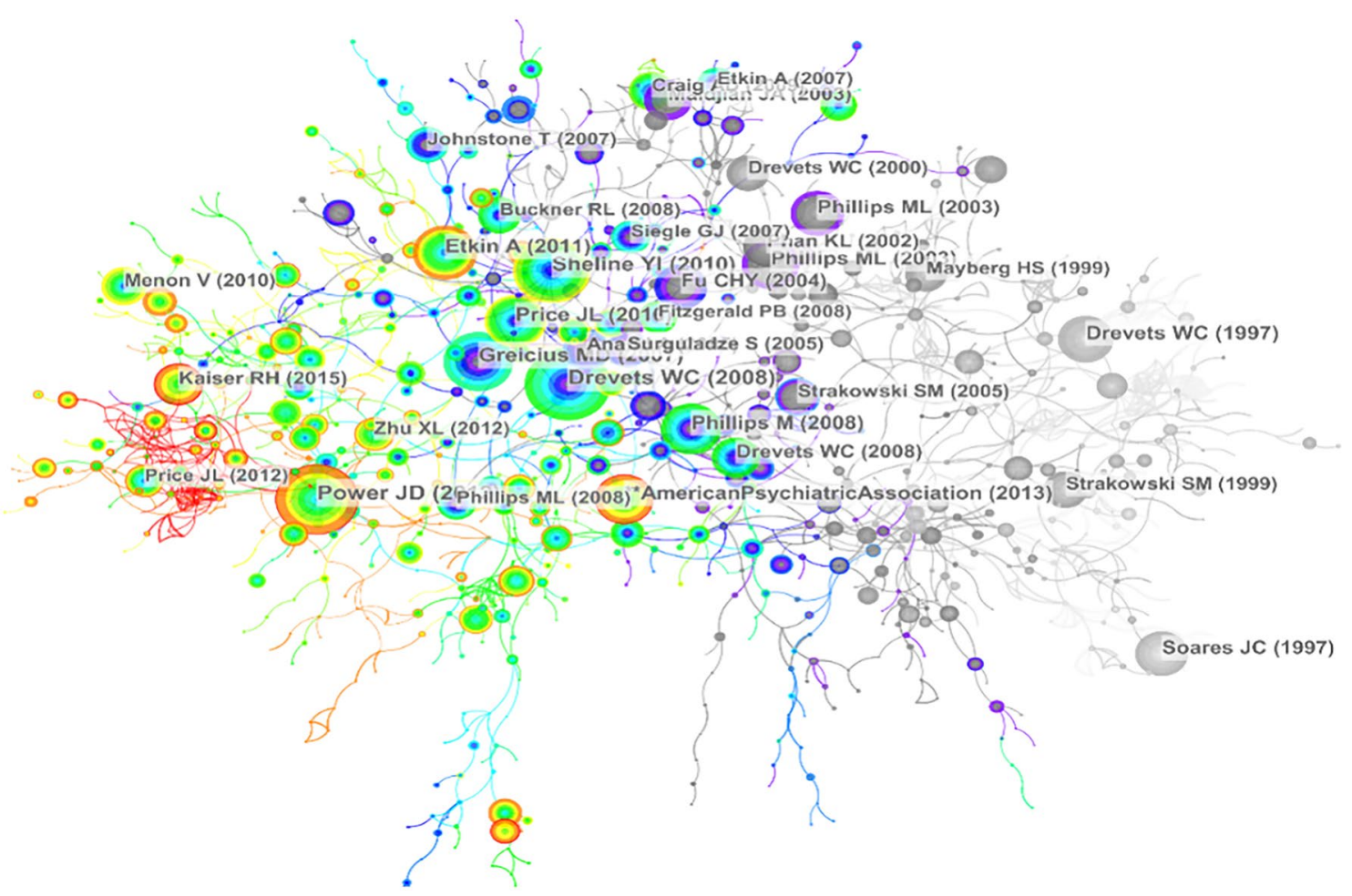

Fig. 8 Reference co-citation map for MRI-associated mood disorder studies 
Table 7 Top 10 cited publications reporting MRI use in mood disorder studies

\begin{tabular}{|c|c|c|c|c|c|c|}
\hline Year & Title & Source & Author & Freq & Burst & Degree \\
\hline 2008 & $\begin{array}{l}\text { Brain Structural and Functional Abnormalities in Mood Disorders: } \\
\text { Implications for Neurocircuitry Models of Depression }\end{array}$ & Brain Struct Funct & Drevets WC & 123 & 28.46 & 2 \\
\hline 2012 & $\begin{array}{l}\text { Spurious but Systematic Correlations in Functional Connectivity } \\
\text { MRI Networks Arise From Subject Motion }\end{array}$ & Neuroimage & Power JD & 121 & 31.96 & 6 \\
\hline 2010 & $\begin{array}{l}\text { Resting-state Functional MRI in Depression Unmasks Increased } \\
\text { Connectivity Between Networks via the Dorsal Nexus }\end{array}$ & P Natl Acad Sci USA & Sheline YI & 112 & 20.51 & 8 \\
\hline 2007 & $\begin{array}{l}\text { Resting-state Functional Connectivity in Major Depression: Abnor- } \\
\text { mally Increased Contributions From Subgenual Cingulate Cortex } \\
\text { and Thalamus }\end{array}$ & Biol Psychiat & Greicius MD & 104 & 24.1 & 4 \\
\hline 2011 & $\begin{array}{l}\text { Emotional Processing in Anterior Cingulate and Medial Prefrontal } \\
\text { Cortex }\end{array}$ & Trends Cogn Sci & Etkin A & 93 & 22.46 & 4 \\
\hline 2010 & Neurocircuitry of Mood Disorders & Neuropsychopharmacol & Price JL & 92 & 15.28 & 3 \\
\hline 2008 & $\begin{array}{l}\text { A Neural Model of Voluntary and Automatic Emotion Regulation: } \\
\text { Implications for Understanding the Pathophysiology and Neurode- } \\
\text { velopment of Bipolar Disorder }\end{array}$ & Mol Psychiatr & Phillips M & 88 & 16.1 & 1 \\
\hline 2003 & $\begin{array}{l}\text { Neurobiology of emotion perception II: implications for major } \\
\text { psychiatric disorders }\end{array}$ & Biol Psychiat & Phillips ML & 86 & 29.2 & 3 \\
\hline 2013 & Diagnostic and Statistical Manual of Mental Disorders (DSM) & Diagn Stat Man Ment & $\begin{array}{l}\text { American Psychi- } \\
\text { atric Associa- } \\
\text { tion }\end{array}$ & 86 & 29.21 & 2 \\
\hline 1997 & Subgenual Prefrontal Cortex Abnormalities in Mood Disorders & Nature & Drevets WC & 83 & 42.41 & 6 \\
\hline
\end{tabular}

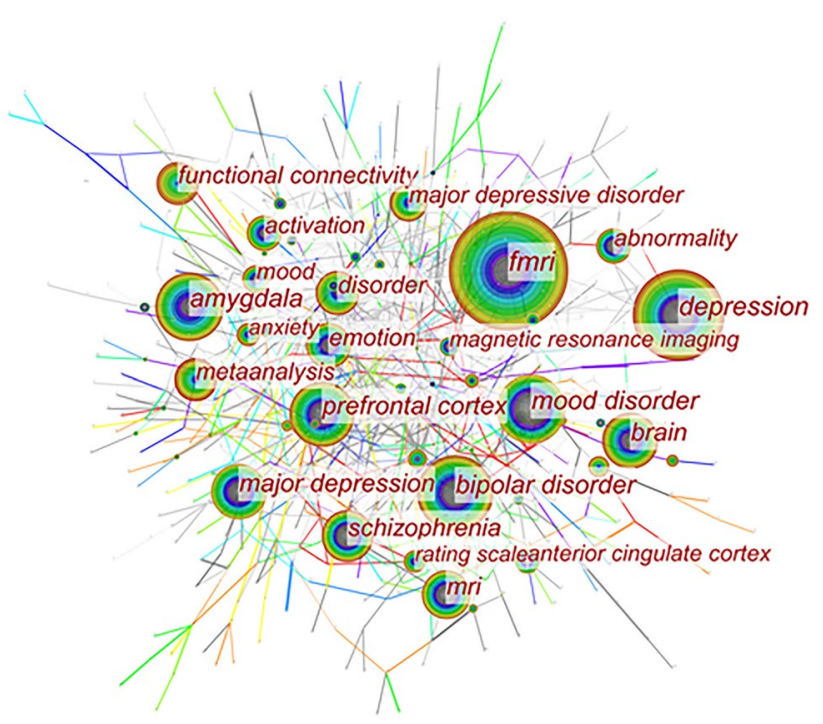

Fig. 9 Keyword co-occurrence map of MRI use in mood disorder studies from 1999 to 2020 . Node size represents keyword co-occurrence frequency. Node size corresponds to keyword frequency

\section{Key brain regions associated with mood disorders}

Neuroimaging is increasingly becoming important in the study of the pathophysiology of mood disorders, with most studies focusing on the amygdala, prefrontal cortex and anterior cingulate cortex. The involvement of amygdala in major depression pathogenesis has been reported in previous studies [36]. While amygdala volume alterations have been implicated in mood disorders [37], Tomas et al. suggested that amygdala volume changes may reflect underlying illness or represent artifacts from medication or comorbidities [38]. The prefrontal cortex (PFC), which is involved in thinking and behavioral regulation has also been implicated in depression [39, 40]. Based on anatomical connectivity and functional specialization, the prefrontal cortex is divided into two subregions: ventromedial prefrontal cortex (vmPFC) and dorsolateral sectors (dlPFC) [41]. The human anterior cingulate cortex (ACC) is involved in response to pain affecting oneself, or others. However, its mechanisms of activity are poorly understood [42]. The mean gray matter volume of the "subgenual" ACC ( $\operatorname{sg}$ ACC) cortex is abnormally low in individuals with major depressive disorder (MDD) and bipolar disorder [43]. Psychopathic traits have been negatively correlated with amygdala-ventral anterior cingulate cortex connectivity for angry versus neutral faces [44]. The anterior cingulate cortex may influence initiation, motivation, and goal-directed behaviors [45].

\section{Research frontiers}

Keyword bursts provide a means of forecasting research direction. CiteSpace $\mathrm{V}$ analysis of burst keywords revealed 209 burst terms and 40 keywords by the end of 2020, and predicted the research hotspots shown in Fig. 10. 
Table 8 Top 20 keywords of magnetic resonance imaging in mood disorders

\begin{tabular}{llllll}
\hline Rank & Keyword & Freq & Rank & Keyword & Freq \\
\hline 1 & fMRI & 862 & 11 & Emotion & 372 \\
2 & Depression & 658 & 12 & Disorder & 365 \\
3 & Bipolar disorder & 570 & 13 & Functional connectivity & 342 \\
4 & Amygdala & 530 & 14 & Metaanalysis & 323 \\
5 & Mood disorder & 517 & 15 & Activation & 317 \\
6 & Prefrontal cortex & 496 & 16 & Abnormality & 312 \\
7 & Brain & 455 & 17 & Major depressive disorder & 301 \\
8 & Major depression & 440 & 18 & Mood & 263 \\
9 & Schizophrenia & 419 & 19 & Anterior cingulate cortex & 233 \\
10 & MRI & 386 & 20 & Magnetic resonance imaging & 221 \\
\hline
\end{tabular}

\section{Discovering abnormal brain structure and network}

In vivo neuroimaging of neural networks that putatively regulate normal emotions have shown that they are implicated in the pathogenesis of mood disorders [12]. Therefore, functional and structural brain pathology may contribute to mood disorders [46, 47]. Reduced gray matter volume has been reported in the posterior cingulate cortex and superior temporal gyrus in bipolar disorder [48], while white matter abnormalities have been associated with major depression [49]. Severe depression cases exhibit ventricular enlargement, sulcal widening, and reduced frontal lobe, hippocampus and caudate nucleus volumes [50]. Advances in brain imaging of mood disorders have been mainly due to studying regional brain abnormalities, and more recently, studying whole brain connectome. Various MRI strategies, including 'functional' MRI, which detects changes in blood flow to a specific area of the brain, blood-oxygen-level-dependent imaging (BOLD) fMRI, which examines changes in oxygenated or deoxygenated blood, and resting state fMRI have significantly improved our knowledge of neuronal activity. Mood disorder patients exhibit morphological abnormalities or morphometry in various visceromotor network structures [51]. Young MDD reported significant reduction in DRNcingulate cortex connectivity relative to healthy controls and a correlation between DRN amygdala/hippocampus complex connectivity and depressive symptom severity [42]. Wayne $\mathrm{C}$ [46] proposed a neural model in which dysfunctions of MPFC and the circuits connecting it to other cortical and limbic structures account for mood disorder associated neural mechanisms.

\section{Sex differences}

Biological differences between sexes affect emotions and social behavior [52]. Lifetime depression and anxiety disorder rates are twice higher in women than men [53, 54]. Gender impacts mood and anxiety disorders and may, therefore, offer some insights into the mechanistic basis of affective disturbances in men and women [55-57]. Besides, women's moods are significantly affected by changes in menstrual cycle. Depression, anxiety and irritability are the three most studied symptoms of PMDD in women of childbearing age [58]. During the perinatal period, mood disturbance are commonly reported by women [59]. Depression is a common complication of pregnancy and the postpartum period [60]. Moreover, menopausal transition is correlated with mood changes ranging from distress to minor depression to major depressive disorders in women [61].The role of reproductive steroids in mood regulation among women may become the most potential research direction [62]. Given that sex differences affect various aspects of the brain, including its structure, function, and stress responses, studies should aim at establishing gender-associated differences that have an effect on mood disorders [63, 64].

\section{Effect of treatment measures on brain activity}

Effective therapies for emotional disorders are urgently needed. Although various pharmacological and non-pharmacological therapeutic approaches, including electroconvulsive therapy, cognitive behavioral therapy, ketogenic diets [65], circadian treatment [66], bright-light therapy [67] and drug naïve (ketamine, lithium salts) [68,69] are available, their efficacies are widely varied between individuals.

\section{Evidence-based medicine with meta-analysis}

Meta-analysis may help discern important findings across disparate studies, thereby, providing actionable information to clinicians [70, 71]. For instance, multiple antidepressants are available for MDD, and their prescription should be informed by the best available data. A meta-analysis by Andrea Cipriani [72] on the relative efficacy and tolerability of 21 antidepressants used to treat major depressive disorders found that all antidepressants were more efficacious than placebo in adults. A meta-analysis study also showed that the relationship between creativity and mood disorders 


\begin{tabular}{|c|c|c|c|c|c|}
\hline Keywords & Year & Strength & Begin & End & $1999-2020$ \\
\hline rumination & 1999 & 4.6713 & 2014 & 2020 & \\
\hline nucleus accumben & 1999 & 3.2264 & 2014 & 2020 & \\
\hline cortical thickness & 1999 & 12.5126 & 2014 & 2020 & \\
\hline network & 1999 & 14.3488 & 2014 & 2020 & \\
\hline comorbidity & 1999 & 3.821 & 2014 & 2020 & = \\
\hline social cognition & 1999 & 4.3748 & 2015 & 2020 & \\
\hline ventral striatum & 1999 & 5.9234 & 2015 & 2020 & E \\
\hline brain network & 1999 & 7.4338 & 2015 & 2020 & $=$ \\
\hline resting-state fmri & 1999 & 9.1527 & 2015 & 2020 & $\underline{=}$ \\
\hline functionalconnectivity & 1999 & 5.3189 & 2015 & 2020 & $=$ \\
\hline neural basis & 1999 & 3.226 & 2015 & 2020 & $=$ \\
\hline cortisol & 1999 & 4.4794 & 2016 & 2020 & 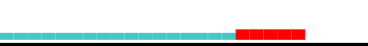 \\
\hline diagnosis & 1999 & 4.5996 & 2016 & 2020 & E \\
\hline state functional connectivity & 1999 & 8.4492 & 2016 & 2020 & 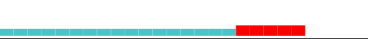 \\
\hline resting state & 1999 & 6.0116 & 2016 & 2020 & E \\
\hline default mode network & 1999 & 17.7588 & 2016 & 2020 & 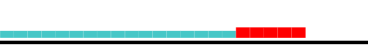 \\
\hline empathy & 1999 & 3.9855 & 2016 & 2020 & E \\
\hline connectivity & 1999 & 7.794 & 2016 & 2020 & $=$ \\
\hline impulsivity & 1999 & 4.7199 & 2016 & 2020 & $\underline{\underline{2}}$ \\
\hline sex difference & 1999 & 3.4206 & 2017 & 2020 & E \\
\hline ptsd & 1999 & 5.8121 & 2017 & 2020 & $\underline{\underline{ }}$ \\
\hline cognitive behavioral therapy & 1999 & 5.0917 & 2017 & 2020 & \\
\hline chronic pain & 1999 & 4.4911 & 2017 & 2020 & $\underline{\underline{\mu}}$ \\
\hline brain structure & 1999 & 3.8852 & 2017 & 2020 & 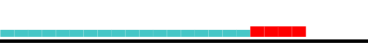 \\
\hline symptom & 1999 & 5.2983 & 2017 & 2020 & $=$ \\
\hline resting state fmri & 1999 & 7.2786 & 2017 & 2020 & $\underline{\underline{E}}$ \\
\hline emotion regulation & 1999 & 11.5739 & 2017 & 2020 & E \\
\hline electroconvulsive therapy & 1999 & 3.514 & 2017 & 2020 & $\underline{\underline{E}}$ \\
\hline drug naive & 1999 & 3.1432 & 2017 & 2020 & $=$ \\
\hline irritability & 1999 & 3.1669 & 2018 & 2020 & $=$ \\
\hline scale & 1999 & 10.2641 & 2018 & 2020 & $=$ \\
\hline segmentation & 1999 & 5.2156 & 2018 & 2020 & $=$ \\
\hline stimulation & 1999 & 3.9361 & 2018 & 2020 & 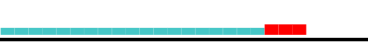 \\
\hline sleep & 1999 & 4.2231 & 2018 & 2020 & $=$ \\
\hline episodic memory & 1999 & 3.3641 & 2018 & 2020 & 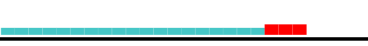 \\
\hline mind & 1999 & 3.6253 & 2018 & 2020 & $=$ \\
\hline trauma & 1999 & 5.8078 & 2018 & 2020 & 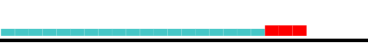 \\
\hline prevalence & 1999 & 7.9204 & 2018 & 2020 & 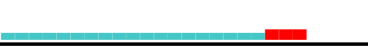 \\
\hline salience network & 1999 & 6.1287 & 2018 & 2020 & 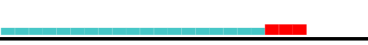 \\
\hline metaanalysis & 1999 & 11.8518 & 2018 & 2020 & 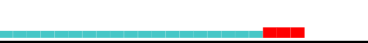 \\
\hline
\end{tabular}

Fig.10 Top 40 Keywords with Citation Bursts from 2016 to 2020 
varied according to the research methodology used [73]. Low doses of partial dopamine agonist therapies represent a relatively safe and effective alternative therapies [74]. Additionally, a meta-analysis showed that lithium, which is commonly used to manage mood disorders, may elevate the risk of low urine concentrations, hypothyroidism, hyperparathyroidism, and weight gain [75].

\section{Conclusions}

We performed a bibliometric analysis of publications involving the use of MRI in mood disorder studies over the last 2 decades. Within this period, the number of such publications rose rapidly. The US, England, and Canada have high publication rates and centrality in this area. Additionally, research collaborations on this topic are very active. Our findings reveal Soares JC as the most prolific author, while the US emerged as the global leader in research investment. Mounting evidence shows that MRI is an effective tool for elucidating neuronal activity. The US department of human health services is the leading funding agency on this topic. We also found that depression, bipolar disorder and schizophrenia are the major focus areas when evaluating major brain regions, including the amygdala, prefrontal cortex and anterior cingulate cortex. Our findings indicate that in future, studies will likely focus on brain structure and network, sex differences, brain changes upon treatment and meta-analyses.

Acknowledgements The authors would like to thank the Research and Innovation team of Emotional Diseases and Syndromes at Shandong University of Traditional Chinese Medicine for their support.

Author contributions GM and SH conceived the study and wrote the manuscript. GM, SH and CX collected and analyzed the data, contributed materials/analysis tools, prepared figures and/or tables and read the final draft. GD and QM contributed to study conception and its coordination. All authors read and approved the final manuscript.

Funding Prof. Mingqi Qiao received funding from National Science \& Technology Major Project "Key New Drug Creation and Manufacturing Program" of China-NO. SQ2017ZX091064, while Prof. Dongmei Gao received funding from the National Natural Science Foundation of China-NO. 81001484 and 81473558.

Data availability The extracted data and statistical syntax are available from the first author upon reasonable request.

\section{Declarations}

Conflicts of interest The authors declare no conflict of interest.

Ethical approval This study is a literature review for which ethical approval was waived.

Informed consent None required.
Open Access This article is licensed under a Creative Commons Attribution 4.0 International License, which permits use, sharing, adaptation, distribution and reproduction in any medium or format, as long as you give appropriate credit to the original author(s) and the source, provide a link to the Creative Commons licence, and indicate if changes were made. The images or other third party material in this article are included in the article's Creative Commons licence, unless indicated otherwise in a credit line to the material. If material is not included in the article's Creative Commons licence and your intended use is not permitted by statutory regulation or exceeds the permitted use, you will need to obtain permission directly from the copyright holder. To view a copy of this licence, visit http://creativecommons.org/licenses/by/4.0/.

\section{References}

1. Rakofsky J, Rapaport M (2018) Mood disorders. Continuum (Minneap Minn) 24:804-827

2. Gordon-Lipkin E, Marvin AR, Law JK, Lipkin PH (2018) Anxiety and mood disorder in children with autism spectrum disorder and ADHD. Pediatrics 141:e20171377

3. Denton EJ et al (2018) Mood disorders are highly prevalent in patients investigated with a multiple sleep latency test. Sleep Breath 22:305-309

4. Uguz F, Yakut E, Aydogan S, Bayman MG, Gezginc K (2019) Prevalence of mood and anxiety disorders during pregnancy: a case-control study with a large sample size. Psychiatry Res 272:316-318

5. Tang MH, Pinsky EG (2015) Mood and affect disorders. Pediatr Rev 36:52-60 (quiz 61)

6. Deligianni F, Guo Y, Yang GZ (2019) From emotions to mood disorders: a survey on gait analysis methodology. IEEE J Biomed Health 23:2302-2316

7. Gruzdev SK, Yakovlev AA, Druzhkova TA, Guekht AB, Gulyaeva NV (2019) The missing link: how exosomes and miRNAs can help in bridging psychiatry and molecular biology in the context of depression, bipolar disorder and schizophrenia. Cell Mol Neurobiol 39:729-750

8. Lovheim H (2012) A new three-dimensional model for emotions and monoamine neurotransmitters. Med Hypotheses 78:341-348

9. Sanders RD, Gillig PM (2010) Gait and its assessment in psychiatry. Psychiatry (Edgmont) 7:38-43

10. Tarlaci S (2019) Quantum neurobiological view to mental health problems and biological psychiatry. J Psychopathol 25:70-84

11. Wilczynska K, Simonienko K, Konarzewska B, Szajda SD, Waszkiewicz N (2018) Morphological changes of the brain in mood disorders. Psychiatr Pol 52:797-805

12. Phillips ML, Drevets WC, Rauch SL, Lane R (2003) Neurobiology of emotion perception II: implications for major psychiatric disorders. Biol Psychiatry 54:515-528

13. Matsumoto K (2016) Development of magnetic resonance-based functional imaging: the past, the present, and the future. Yakugaku Zasshi 136:1075-1080

14. El-Serougy L, Abdel Razek AAK, Ezzat A, Eldawoody H, ElMorsy A (2016) Assessment of diffusion tensor imaging metrics in differentiating low-grade from high-grade gliomas. Neuroradiol J 29:400-407

15. Abdel Razek AAK, Talaat M, El-Serougy L, Gaballa G, Abdelsalam M (2019) Clinical applications of arterial spin labeling in brain tumors. J Comput Assist Tomogr. 43:525-532

16. Razek AAKA, Abdalla A, Ezzat A, Megahed A, Barakat T (2014) Minimal hepatic encephalopathy in children with liver 
cirrhosis: diffusion-weighted MR imaging and proton MR spectroscopy of the brain. Neuroradiology 56:885-891

17. Tulay EE, Metin B, Tarhan N, Arıkan MK (2019) Multimodal neuroimaging: basic concepts and classification of neuropsychiatric diseases. Clin EEG Neurosci 50:20-33

18. Ai $\mathrm{T}$ et al (2012) A historical overview of magnetic resonance imaging, focusing on technological innovations. Invest Radiol 47:725-741

19. Mathias LK, Monette PJ, Harper DG, Forester BP (2017) Application of magnetic resonance spectroscopy in geriatric mood disorders. Int Rev Psychiatry 29:597-617

20. Lai C-H (2019) The neural markers of MRI to differentiate depression and panic disorder. Prog Neuro-Psychoph 91:72-78

21. Petersen N, Ghahremani DG, Rapkin AJ et al (2019) Restingstate functional connectivity in women with PMDD. Transl Psychiatry 9(1):339

22. Budinger TF, Bird MD (2018) MRI and MRS of the human brain at magnetic fields of $14 \mathrm{~T}$ to $20 \mathrm{~T}$ : technical feasibility, safety, and neuroscience horizons. Neuroimage 168:509-531

23. Wang L, Xia E, Li H et al (2019) A bibliometric analysis of crowdsourcing in the field of public health. Int J Environ Res Public Health 16(20)

24. Liang YD et al (2017) Study of acupuncture for low back pain in recent 20 years: a bibliometric analysis via CiteSpace. J Pain Res 10:951-964

25. Chen CM, Hu ZG, Liu SB, Tseng H (2012) Emerging trends in regenerative medicine: a scientometric analysis in CiteSpace. Expert Opin Biol Ther 12:593-608

26. Chen C (2016) CiteSpace: a practical guide for mapping scientific literature

27. Bang CS, Lee JJ, Baik GH (2019) The most influential articles in Helicobacter pylori research: a bibliometric analysis. Helicobacter 24:e12589

28. Huang X, Fan X, Ying J, Chen S (2019) Emerging trends and research foci in gastrointestinal microbiome. J Transl Med 17:67

29. Farrokhyar $F$ et al (2016) Impact of research investment on scientific productivity of junior researchers. Transl Behav Med 6:659-668

30. Squitieri L, Chung KC (2014) Funding research in the twentyfirst century: current opinions and future directions. Hand clin 30(3):367-376

31. Wormser GP, Hassett AL (2019) Depression in the primary care setting. N Engl J Med 380:2278-2279

32. Krishnan V, Nestler EJ (2008) The molecular neurobiology of depression. Nature 455:894-902

33. Anthes E (2014) Depression: a change of mind. Nature 515:185-187

34. Anderson IM, Haddad PM, Scott J (2012) Bipolar disorder. BMJ 345:e8508

35. Tandon $\mathrm{R}$ et al (2013) Definition and description of schizophrenia in the DSM-5. Schizophr Res 150:3-10

36. Drevets WC (2003) Neuroimaging abnormalities in the amygdala in mood disorders. Ann N Y Acad Sci 985:420-444

37. Velakoulis D et al (2006) Hippocampal and amygdala volumes according to psychosis stage and diagnosis: a magnetic resonance imaging study of chronic schizophrenia, first-episode psychosis, and ultra-high-risk individuals. Arch Gen Psychiatry 63:139-149

38. Hajek $\mathrm{T}$ et al (2009) Amygdala volumes in mood disorders-metaanalysis of magnetic resonance volumetry studies. J Affect Disord 115:395-410

39. Treadway MT et al (2015) Illness progression, recent stress, and morphometry of hippocampal subfields and medial prefrontal cortex in major depression. Biol Psychiatry 77:285-294

40. Liu W et al (2017) The role of neural plasticity in depression: from hippocampus to prefrontal cortex. Neural Plast 2017:6871089
41. Koenigs M, Grafman J (2009) The functional neuroanatomy of depression: distinct roles for ventromedial and dorsolateral prefrontal cortex. Behav Brain Res 201:239-243

42. Carrillo M et al (2019) Emotional mirror neurons in the rat's anterior cingulate cortex. Curr Biol 29:2104

43. Drevets WC, Savitz J, Trimble M (2008) The subgenual anterior cingulate cortex in mood disorders. CNS Spectr 13:663-681

44. Ewbank MP et al (2018) Psychopathic traits influence amygdalaanterior cingulate cortex connectivity during facial emotion processing. Soc Cogn Affect Neurosci 13:525-534

45. Devinsky O, Morrell MJ, Vogt BA (1995) Contributions of anterior cingulate cortex to behaviour. Brain 118(Pt 1):279-306

46. Drevets WC, Price JL, Furey ML (2008) Brain structural and functional abnormalities in mood disorders: implications for neurocircuitry models of depression. Brain Struct Funct 213:93-118

47. Anand A (2019) Editorial: functional and structural connectomics of mood disorders. Front Psychiatry 10:202

48. Nugent AC et al (2006) Cortical abnormalities in bipolar disorder investigated with MRI and voxel-based morphometry. Neuroimage 30:485-497

49. Tham MW, Woon PS, Sum MY, Lee TS, Sim K (2011) White matter abnormalities in major depression: evidence from post-mortem, neuroimaging and genetic studies. J Affect Disord 132:26-36

50. Schweitzer I, Tuckwell V, Ames D, O'Brien J (2001) Structural neuroimaging studies in late-life depression: a review. World $\mathrm{J}$ Biol Psychiatry 2:83-88

51. Drevets WC (2001) Neuroimaging and neuropathological studies of depression: implications for the cognitive-emotional features of mood disorders. Curr Opin Neurobiol 11:240-249

52. Choleris E, Galea LAM, Sohrabji F, Frick KM (2018) Sex differences in the brain: implications for behavioral and biomedical research. Neurosci Biobehav Rev 85:126-145

53. Weissman MM et al (1996) Cross-national epidemiology of major depression and bipolar disorder. JAMA 276:293-299

54. Gater R et al (1998) Sex differences in the prevalence and detection of depressive and anxiety disorders in general health care settings: report from the World Health Organization Collaborative Study on Psychological Problems in General Health Care. Arch Gen Psychiatry 55:405-413

55. Hirsh AT, Waxenberg LB, Atchison JW, Gremillion HA, Robinson ME (2006) Evidence for sex differences in the relationships of pain, mood, and disability. J Pain 7:592-601

56. Hankin BL (2009) Development of sex differences in depressive and co-occurring anxious symptoms during adolescence: descriptive trajectories and potential explanations in a multiwave prospective study. J Clin Child Adolesc Psychol 38:460-472

57. Rainville JR, Hodes GE (2019) Inflaming sex differences in mood disorders. Neuropsychopharmacology 44:184-199

58. Ko CH et al (2013) Depression, irritability, and anxiety in women with premenstrual dysphoric disorder. Int J Psychiatry Med 46:39-55

59. Bei B, Coo S, Trinder J (2015) Sleep and mood during pregnancy and the postpartum period. Sleep Med Clin 10:25-33

60. Becker M, Weinberger T, Chandy A, Schmukler S (2016) Depression during pregnancy and postpartum. Curr Psychiatry Rep 18:32

61. Alexander JL et al (2007) Neurobehavioral impact of menopause on mood. Expert Rev Neurother 7:S81-S91

62. McEvoy K, Osborne LM (2019) Allopregnanolone and reproductive psychiatry: an overview. Int Rev Psychiatry 31:237-244

63. Altemus M, Sarvaiya N, Neill Epperson C (2014) Sex differences in anxiety and depression clinical perspectives. Front Neuroendocrinol. 35:320-330

64. Rubinow DR, Schmidt PJ (2019) Sex differences and the neurobiology of affective disorders. Neuropsychopharmacology 44:111-128 
65. Brietzke E et al (2018) Ketogenic diet as a metabolic therapy for mood disorders: Evidence and developments. Neurosci Biobehav Rev 94:11-16

66. Huhne A, Welsh DK, Landgraf D (2018) Prospects for circadian treatment of mood disorders. Ann Med 50:637-654

67. Pail G et al (2011) Bright-light therapy in the treatment of mood disorders. Neuropsychobiology 64:152-162

68. Haussmann R, Lewitzka U, Severus E, Bauer M (2017) Correct treatment of mood disorders with lithium. Nervenarzt 88:1323-1334

69. Sanacora G et al (2017) A consensus statement on the use of ketamine in the treatment of mood disorders. JAMA Psychiat 74:399-405

70. Paul M, Leibovici L (2014) Systematic review or meta-analysis? Their place in the evidence hierarchy. Clin Microbiol Infect 20:97-100

71. Lee YH (2018) An overview of meta-analysis for clinicians. Korean J Intern Med 33:277-283
72. Cipriani A et al (2018) Comparative efficacy and acceptability of 21 antidepressant drugs for the acute treatment of adults with major depressive disorder: a systematic review and network metaanalysis. Lancet 391:1357-1366

73. Taylor CL (2017) Creativity and Mood disorder: a systematic review and meta-analysis. Perspect Psychol Sci 12:1040-1076

74. Romeo B, Blecha L, Locatelli K, Benyamina A, Martelli C (2018) Meta-analysis and review of dopamine agonists in acute episodes of mood disorder: efficacy and safety. J Psychopharmacol 32:385-396

75. McKnight RF et al (2012) Lithium toxicity profile: a systematic review and meta-analysis. Lancet 379:721-728

Publisher's Note Springer Nature remains neutral with regard to jurisdictional claims in published maps and institutional affiliations. 